\title{
OÚRICURI
}

\section{AVALIAÇÃO DA QUALIDADE DE VIDA EM IDOSOS MATRICULADOS EM UM PROGRAMA UNIVERSITÁRIO PARA IDOSOS}

\author{
Everton BOFF ${ }^{1 *}$; Taíne GREGORY² \\ ${ }^{1 *}$ Mestre em Ciências Farmacêuticas. Universidade do Oeste de Santa Catarina. *Autor correspondente. E- \\ mail: everton.boff@unoesc.edu.br \\ 2Psicóloga. Universidade do Oeste de Santa Catarina. E-mail: tainegregory@hotmail.com \\ Recebido: 29.03.2019 Aceito: 17.06.2019 \\ http://doi.org/10.29327/ouricuri.v9.i1.a3
}

\begin{abstract}
Resumo: O envelhecimento populacional em alguns países, como no Brasil é notório, é caracterizado por um aumento considerável da população acima de 60 anos. Esse fenômeno vem demandando novos tratamentos e cuidados para a manutenção de uma boa saúde, considerando o envelhecimento como um processo fisiológico natural na vida das pessoas. Entretanto, muitos indivíduos da terceira idade não compreendem tais recomendações e necessitam de cuidados específicos de saúde, o que leva profissionais da saúde demandarem pesquisas e consequentes entendimentos dessa fase tão importante da vida humana. Este trabalho objetivou estudar a presença de doenças crônicas não transmissíveis e a polimedicação em idosos que frequentam a Universidade da Terceira Idade (UNITI) da Universidade do Oeste de Santa Catarina (UNOESC), assim como o apoio familiar à mesma. Adicionalmente, investigou a presença de depressão geriátrica, bem como a capacidade funcional, cognitiva e perceptiva dos idosos sobre a própria qualidade de vida, em diversos âmbitos. Os resultados encontrados demonstram que a população idosa entrevistada é portadora de doenças crônicas não transmissíveis e depressão leve, com pouco comprometimento de suas capacidades funcionais e cognitivas. Apresentam boa qualidade de vida, considerando os âmbitos físico, psicológico, social e a relação com o meio ambiente. Diante disso, conclui-se que os alunos da UNITI, programa mantido pela UNOESC, apresentam dados semelhantes com a realidade nacional em alguns aspectos, porém, melhores resultados em outros, demonstrando um nível de qualidade de vida superior ao da maioria da população brasileira pertencente a essa faixa etária, confirmando que o aprendizado em sala de aula está sendo colocado em prática pelos idosos, refletindo em melhor qualidade de vida.
\end{abstract}

Palavras-chave: Saúde; Estilo de vida; Doenças crônicas; Terceira idade.

\section{EVALUATION OF QUALITY OF LIFE IN ELDERLY ENROLLMENT IN A UNIVERSITY PROGRAM FOR ELDERLY}

Abstract: Population aging in some countries, as in Brazil is notorious, is characterized by a considerable increase in population over 60 years. This phenomenon demands new treatments and care for the maintenance of good health, considering aging as a natural physiological process in people's lives. However, many seniors do not understand these recommendations and need specific health care, which leads health professionals to demand research and consequent understandings of this important phase of human life. This study aimed to study the presence of noncommunicable chronic diseases and the polymedication in elderly people attending the University of the Third Age (UNITI) of the University of the West of Santa Catarina (UNOESC), as well as the family support. Additionally, it investigated the presence of geriatric depression, as well as the functional, cognitive and perceptive capacity of the elderly about the quality of life itself, in several areas. The results show that the elderly population interviewed are carriers of chronic noncommunicable diseases and

Revista Ouricuri, Juazeiro, Bahia, v.9, n.1. p.027-041. jan./jun., 2019.

http://www.revistas.uneb.br/index.php/ouricuri | ISSN 2317-0131 
mild depression, with little impairment of their functional and cognitive abilities. They present good quality of life, considering the physical, psychological, social and the environment. Thus, it is concluded that UNITI students, a program maintained by UNOESC, present similar data with the national reality in some aspects, but better results in others, demonstrating a higher level of quality of life than the majority of the Brazilian population belonging to to this age group, confirming that the learning in the classroom is being put into practice by the elderly, reflecting in a better quality of life.

Key words: Health; Lifestyle; Chronic diseases; Third Age.

\section{EVALUACIÓN DE LA CALIDAD DE VIDA EN LOS ANCIANOS MATRICULADOS EN UN PROGRAMA UNIVERSITARIO PARA ANCIANOS}

Resumen: El envejecimiento poblacional en algunos países, como en Brasil es notorio, se caracteriza por un aumento considerable de la población de más de 60 años. Este fenómeno viene demandando nuevos tratamientos y cuidados para el mantenimiento de una buena salud, considerando el envejecimiento como un proceso fisiológico natural en la vida de las personas. Sin embargo, muchos individuos de la tercera edad no entienden tales recomendaciones y necesitan cuidados específicos de salud, lo que lleva a profesionales de la salud demandar investigaciones y consecuentes entendimientos de esa fase tan importante de la vida humana. Este trabajo tuvo como objetivo estudiar la presencia de enfermedades crónicas no transmisibles y la polimedicación en ancianos que frecuentan la Universidad de la Tercera Edad (UNITI) de la Universidad del Oeste de Santa Catarina (UNOESC), así como el apoyo familiar a la misma. Adicionalmente, investigó la presencia de depresión geriátrica, así como la capacidad funcional, cognitiva y perceptiva de los ancianos sobre la propia calidad de vida, en diversos ámbitos. Los resultados encontrados demuestran que la población anciana entrevistada es portadora de enfermedades crónicas no transmisibles y depresión leve, con poco comprometimiento de sus capacidades funcionales y cognitivas. Presentan buena calidad de vida, considerando los ámbitos físico, psicológico, social y la relación con el medio ambiente. En este sentido, se concluye que los alumnos de UNITI, programa mantenido por la UNOESC, presentan datos semejantes con la realidad nacional en algunos aspectos, sin embargo, mejores resultados en otros, demostrando un nivel de calidad de vida superior al de la mayoría de la población brasileña perteneciente a ese grupo de edad, confirmando que el aprendizaje en el aula está siendo puesto en práctica por los ancianos, reflejando en mejor calidad de vida.

Palabras clave: Salud; Estilo de vida; Enfermedades crónicas; Tercera edad.

\section{INTRODUÇÃO}

O envelhecimento populacional é uma realidade presente e crescente no Brasil e no mundo (Veras, 2009). É um processo dinâmico e inerente a todos os seres humanos (Papaléo Neto, 2007). "A velhice é o último período da evolução natural da vida e caracteriza-se por um conjunto de parâmetros biológicos, econômicos, políticos e sociais que compõem o cotidiano das pessoas que vivem esta fase" (Silva et al., 2010).

No Brasil, o "Estatuto do Idoso" referenda que idosa é a pessoa com idade igual ou superior a 60 anos (Brasil, 2003), levando em consideração esta legislação, o critério cronológico também é adotado nos trabalhos científicos, por causa da dificuldade de se estabelecer critérios biológicos (Papaléo Neto, 2007). A idade cronológica mensura somente o número de anos decorridos desde o nascimento da pessoa, não é um índice isolado de desenvolvimento biológico, psicológico e social, pois existem muitos outros fatores que congregam para o conjunto de desenvolver e envelhecer de cada indivíduo (Schneider e Irigaray, 2008)

Revista Ouricuri, Juazeiro, Bahia, v.9, n.1. p.027-041. jan./jun., 2019.

http://www.revistas.uneb.br/index.php/ouricuri | ISSN 2317-0131 
Pensando no processo de envelhecer, as doenças e incapacidades nos idosos, dentre outras questões, podem contribuir para o seu isolamento social, pois a maioria apresenta uma diminuição na sua participação comunitária, social e alguns até mesmo familiares (Geib, 2012), logo, as doenças e os medicamentos estão presentes no cotidiano das pessoas idosas, sendo a polimedicação uma prática comum nesta população (Brasil, 2006).

Conforme Ferreira (2010), os idosos constituem 50\% dos sujeitos que utilizam múltiplos medicamentos. A polimedicação é definida como o uso de vários medicamentos de forma simultânea. Ainda que não exista um consenso na literatura quanto ao número mínimo de medicamentos para classificar a polimedicação vários autores a consideram enquanto uso concomitante de cinco ou mais medicamentos (Flores e Mengue, 2005; Rozenfeld et al., 2008; Secoli, 2010; Carvalho et al., 2012; Neves et al., 2013).

A ocorrência da polimedicação pode ser explicada pelo número de doenças crônicas que acometem os idosos, elevada incidência de sintomas e a realização de consulta e tratamento com especialistas diferentes (Brasil, 2006). O fácil acesso a medicamentos e a baixa frequência do uso de recursos não farmacológicos para a recuperação da saúde também contribuem para o consumo elevado de medicamentos pela população idosa (Flores e Mengue, 2005; Silva et al., 2012).

A polimedicação, aliada a presença de múltiplas doenças, alterações fisiológicas do idoso e o atendimento por vários médicos, torna o idoso mais suscetível ao uso de medicamentos potencialmente inapropriados (Ribas e Oliveira, 2014). "Racionalizar o uso de medicamentos e evitar os agravos advindos da polifarmácia e da iatrogenia serão, sem dúvida, um dos grandes desafios da saúde pública desse século" (Brasil, 2006). Diante disso, observa-se uma necessidade de mudança no modelo de cuidado a população idosa, com ações diferenciadas e efetivas, com o olhar para a integralidade do cuidado, visando acrescentar qualidade nos anos adicionais vividos pelos idosos (Marcon, 2016).

Capacidade funcional é entendida como a autonomia para realizar atividades diárias básicas como comer, tomar banho, escovar os dentes e vestir-se, sem precisar da ajuda de outras pessoas, sendo influenciada por fatores demográficos, socioeconômicos, culturais e psicossociais. Quando esta capacidade está comprometida a capacidade funcional e a carga recai para a família e para o sistema de saúde (ROSA, 2003).

Normalmente, a capacidade funcional é classificada como presente ou ausente, porém na verdade é medida em grau de limitação, desde um grau leve até muito severa onde então o indivíduo passa ser completamente dependente de um cuidador (Parahyba et al., 2005). A saúde do idoso está diretamente relacionada com a manutenção da sua funcionalidade. O idoso que, apesar de apresentar uma ou mais doenças crônicas, mantém suas habilidades físicas e mentais necessárias para uma vida independente e autônoma, deve ser considerado um idoso saudável, pois o mesmo consegue desempenhar normalmente suas atividades e manter uma boa qualidade de vida (Veras, 2009). 
Durante o processo de envelhecimento ocorre a diminuição de algumas funções cognitivas, como aprendizagem e memória. Na maioria das vezes o declínio no funcionamento cognitivo é provocado pelo desuso (falta de prática), doenças (como depressão), fatores comportamentais (como consumo de álcool e medicamentos), fatores psicológicos (por exemplo, falta de motivação, de confiança e baixas expectativas) e fatores sociais (como solidão e isolamento), mais do que o envelhecimento (Schneider e Irigaray, 2008).

Nos últimos anos estamos presenciando a transição demográfica, com o envelhecimento populacional devido principalmente a longevidade, o que demanda um avanço não somente na questão de medicamentos, mas principalmente em tratamentos psicológicos, como por exemplo, a psicoterapia que pode ser fundamental para trabalhar as angústias que podem estar vivenciando nesta fase, sendo estas, a mudanças da estrutura familiar, ansiedade, morte, luto, medo da dependência e declínio cognitivo (Costa, 1998).

Este processo de acordo com a perspectiva psicodinâmica (Costa, 1998) chamada de envelhecimento está em acordo com alguns conflitos e desejos não satisfeitos, desenvolvimento de inferioridade, reminiscências positivas e negativas, tensão narcisistas e chegando sem cuidados a identificação com o negativo do ideal do eu e assim cabendo ao profissional da saúde entender e identificar os cuidados necessários para tornar esta fase da vida leve e sem muitos problemas na sintomatologia psíquica.

A fase de envelhecimento demanda cuidados especiais, que segundo Altman (2011) vai desde a saúde mental, até a atenção com alimentação, práticas de atividades físicas e cuidados com medicamentos, sendo que estes cuidados podem contribuir para a ausência de doenças mentais, influenciando na qualidade de vida dessas pessoas para que possam compreender a grandiosidade desta fase da vida, por mais que sejam identificados restrições psicológicas, mentais ou funcionais, a tecnologia e processos psicoterápicos como forma de ajuda na superação de problemas vem sendo utilizados para comportar todas estas imposições atribuídas aos idosos (Fonseca, 2010).

Nesta fase os idosos estão vivenciando uma infinidade de sentimentos durante seus dias, sendo eles, alegria, amor, satisfação, tristeza, raiva e frustração. Ao mesmo tempo, enfrentam diversos desafios, necessitando de equilíbrio emocional para conseguir enfrentar e passar por estas etapas e empecilhos como confusões, agitações, traumas ou mudanças enormes nos diferentes períodos da vida (Fonseca, 2010).

Para a Organização Mundial de Saúde (OMS), a qualidade de vida é definida como "a percepção do indivíduo sobre a sua posição na vida, no contexto da cultura e dos sistemas de valores nos quais ele vive, e em relação a seus objetivos, expectativas, padrões e preocupações (WHO, 1995)". Na terceira idade é necessário ter qualidade de vida o que está fortemente ligada a uma condição de saúde dos idosos que lhes permita fazer as atividades e desafios que desejam e vão passar, durante o máximo de tempo possível, pois envelhecer não significa parar, mas sim 
acreditar e lutar que se pode ir ainda mais longe que podemos, pois com o aumento da expectativa de vida, ter uma condição física se torna ainda mais relevante para estar preparado para ultrapassar todos os desafios da vida (Rikli e Jones, 2008).

A transição demográfica vivida por todos os países trouxe a necessidade de se pensar em espaços para os idosos que lhes garantissem integração social, para isso como já citada, surgem as Universidades Abertas para a Terceira Idade. As primeiras surgiram na França por volta de 1960, e no Brasil a partir da década de 1990. Estas instituições visam promover a melhora da autoestima, resgate da cidadania, incentivar a autonomia, a independência e reinserção social em busca de uma velhice com mais qualidade de vida (Silva, 2008).

Neste sentido, as Universidades Abertas oferecem algumas oportunidades de atividades para estes idosos, como palestras, teatro, dança jogos, ginástica entre outros, que ao longo dos anos puderam comprovar seus benefícios na vida destas pessoas. Assim, o espaço das Universidades Abertas se constitui como um excelente local para o convívio e interação de idosos, onde podem dialogar com jovens, adultos, alunos da universidade, desenvolvendo esse modelo de ensino e prevenção de doenças neurodegenerativas, já que beneficiam e incentivam participação social e atividades funcionais e cognitivas (Lopes, 2012).

Neste contexto, emerge a seguinte situação problema: Os idosos, participantes ativos da UNITI, apresentam uma melhora no perfil do uso de medicamentos, polimedicação, percepção de qualidade de vida, capacidade cognitiva e funcional? Assim a presente pesquisa objetivou analisar a polimedicação, qualidade de vida, capacidade funcional e cognitiva em idosos participantes a mais de um semestre letivo das aulas da UNITI nos municípios de Maravilha, Pinhalzinho, São José do Cedro e São Miguel do Oeste/SC.

\section{MATERIAL E MÉTODOS}

Trata-se de uma pesquisa de abordagem quantitativa, com cunho descritivo e corte transversal. A pesquisa se deu com 142 idosos, de ambos os sexos matriculados na UNITI dos quatros campi vinculados a UNOESC de São Miguel do Oeste (SC), que são eles: Maravilha, Pinhalzinho, São José do Cedro e São Miguel do Oeste. Os idosos matriculados tinham uma assiduidade de, no mínimo $75 \%$ nas atividades de aulas disponibilizadas até o momento da aplicação dos questionários.

A amostra foi estratificada por sexo e tempo de ingresso na UNITI. Como critério de inclusão, foi levado em consideração a seleção da UNOESC que denomina a UNITI como curso de extensão voltado para pessoas com idade acima de 55 anos, bem como, a avaliação da OMS, a qual considera idosos, pessoas acima de 60 anos de idade para países em desenvolvimento e com ou mais de 65 anos para países desenvolvidos. Sendo assim, inclusos nesta pesquisa todos os idosos acima de 60 anos de idade matriculados na UNITI. 
Para avaliação multidimensional do idoso foi utilizado o questionário adaptado de Morais (2007). Esse questionário foi construído a partir de questões validadas do Projeto Saúde, Bem-estar e Envelhecimento na América Latina e Caribe (SABE) da Organização Pan-americana de Saúde (OPAS), constando questões relacionadas a informações pessoais, condições de moradia, composição familiar, uso de medicamentos, condições de saúde e hábitos de vida, uso e acesso aos serviços de saúde e apoio familiar recebido. Para esse estudo consideramos polimedicados os idosos que utilizam cinco ou mais medicamentos (Flores e Mengue, 2005).

A condição socioeconômica foi avaliada através dos Critérios de Classificação Econômica da Associação Brasileira de Empresas de Pesquisa (ABEP). Esses critérios são baseados em um instrumento de segmentação econômica que utiliza o levantamento de características domiciliares (presença e quantidade de alguns itens domiciliares de conforto e grau de escolaridade do chefe de família) para diferenciar a população (ABEP, 2015).

Também foi utilizada a Escala de Depressão Geriátrica de Yesavage - versão reduzida (GDS - 15), que é um instrumento validado para diagnóstico de depressão em pacientes idosos. É um teste para detecção de sintomas depressivos no idoso, com 15 perguntas afirmativas/negativas. A cada resposta afirmativa, soma-se 1 ponto. Uma pontuação de 0 a 5 se considera normal, 6 a 10 indica depressão leve e o escore maior ou igual a 11 caracteriza depressão grave (Brasil, 2006; Ferrari e Dalacorte, 2007).

O WHOQOL Breaf utilizou-se para mensurar a qualidade de vida relacionada à saúde em grupos de indivíduos enfermos, sadios e idosos, ele é composto por quatro domínios da qualidade de vida, sendo que cada domínio tem por objetivo analisar respectivamente, a capacidade física, o bem-estar psicológico, as relações sociais e o meio ambiente onde o indivíduo está inserido, cada domínio é composto por questões, cujas pontuações de respostas variam de um a cinco. Os escores finais de cada domínio foram calculados por uma sintaxe, que considera as respostas de cada questão que compõe o domínio, resultando em escores finais numa escala de 4 a 20, que podem ser transformados em escala de 0 a 100 (Pereira et al., 2006).

A capacidade funcional foi avaliada por meio do Índice de Barthel (Mahoney e Barthel,1965) e da Escala de Lawton (1983). O Índice de Barthel considera o nível de capacidade funcional para realização das Atividades Básicas da Vida Diária (ABVD), que são tarefas de autocuidado, como banhar-se, alimentar-se ou vestir-se. Já a escala de Lawton avalia o nível de dependência para realização das Atividades Instrumentais da Vida Diária (AIVD), complementando a avaliação obtida com o Índice de Barthel (Lawton, 1983). Ela aborda questões que avaliam o nível de dependência para a realização de atividades mais complexas, como uso do telefone, preparo das refeições, realização de compras, viagens, trabalhos domésticos, uso de medicamentos e manuseio de dinheiro.

Os níveis de atividade física foram avaliados pelo Questionário Internacional de Atividade Física (IPAQ) composto por perguntas relacionadas à frequência e duração de caminhadas, 
atividades cotidianas que exigem esforços físicos de intensidades moderada e vigorosa, além do tempo despendido em atividades executadas em posição sentada.

Após termos a permissão do professor, a coleta de dados foi obtida através de entrevistas realizadas durante as aulas. Cada indivíduo selecionado foi previamente informado sobre os objetivos e procedimentos da pesquisa, posteriormente, cessada todas as dúvidas, concordando em participar, o mesmo era direcionado para um local apropriado onde, o idoso ou representante legal assinava o Termo de Consentimento Livre e Esclarecido para após, responder os instrumentos de avaliação sendo preservada em todos os momentos da pesquisa a identidade do participante.

Para a realização da pesquisa, primeiramente foi solicitado autorização da Coordenação da Universidade da Terceira Idade. Após, o presente trabalho foi encaminhado para avaliação e posterior aprovação pelo Comitê de Ética em Pesquisas (CEP) da Universidade do Oeste de Santa Catarina (UNOESC), tendo os seguintes protocolos de aprovação 1.973.466, 2.040.273 e 2.578.806.

\section{RESULTADOS}

Na pesquisa foram abordados aproximadamente 270 idosos que constituem 9 turmas da Universidade de Terceira Idade dos campi de Maravilha, Pinhalzinho, São José do Cedro e São Miguel do Oeste. Destes, apenas 163 aceitaram responder o questionário apresentado. No entanto, foi considerado, exclusivamente, 142 questionários devido aos outros 21 estarem incompletos. A partir da união e análise dos dados sociais obtidos, é apresentado o perfil da população estudada quando ao sexo, idade, grau de escolaridade de estudo, aposentadoria e valor do benefício (Tabela 1).

Nos últimos anos, a longevidade está aumentando, o que demanda manter um estilo de vida ativo, saudável e flexível para se ter mais qualidade de vida e independência. Sendo possível assim, amenizar ou até mesmo prevenir algumas doenças características nessa fase da vida (WHO, 2005).

Analisando a qualidade de vida dos idosos e o contexto abordado na tabela 1 podemos verificar que $87 \%$ da amostragem da pesquisa são mulheres, levando-nos a considerar que esse índice, diz respeito a sua expectativa de vida, que é de cinco a oito vezes maiores do que a dos homens. Além de maior participação entre os gêneros, as mulheres idosas apresentam maior disposição para atividades em grupos, devido a sua facilidade de socialização (Aires et al., 2015).

De encontro com o dado exposto, há autores que associam esse fato à própria cultura masculina, em que, o cuidado não é visto como uma prática vinculada ao perfil, levando-nos a refletir sobre a importância de analisarmos a situação, e averiguarmos quais as resistências existentes neste contexto (Araújo Gomes e Nascimento, 2007). Aos olhos do sexo oposto, a busca pela identidade e pela valorização da memória está sempre associada com o bem-estar e qualidade de vida, sendo que esta pode ser afirmada pelos dados ligados a qualidade de vida aferida pelos participantes, onde 50\% estão satisfeitos (Ribeiro, 2007). 
Tabela 1. Perfil da população estudada quando ao sexo, idade, grau de escolaridade, aposentadoria e valor do benefício.

\begin{tabular}{|c|c|c|}
\hline Sexo & Número Absoluto & Número Relativo (\%) \\
\hline Feminino & 126 & 88,73 \\
\hline Masculino & 16 & 11,26 \\
\hline Idade & Número Absoluto & Número Relativo (\%) \\
\hline 53 anos ou menos & 05 & 3,52 \\
\hline 54 a 59 anos & 25 & 17,60 \\
\hline 60 a 65 anos & 51 & 35,91 \\
\hline 66 a 71 anos & 39 & 27,46 \\
\hline 72 a 77 anos & 18 & 12,67 \\
\hline 78 anos ou mais & 04 & 2,81 \\
\hline Grau de escolaridade & Número Absoluto & Número Relativo (\%) \\
\hline Analfabeto & -- & -- \\
\hline Ensino Fund. Incompleto & 47 & 33,09 \\
\hline Ensino Fund. Completo & 13 & 9,15 \\
\hline Ensino Médio Incompleto & 16 & 11,26 \\
\hline Ensino Médio Completo & 33 & 23,23 \\
\hline Ensino Sup. Incompleto & 05 & 3,52 \\
\hline Ensino Sup. Completo & 24 & 16,90 \\
\hline Pós-Graduado & 04 & 2,81 \\
\hline Aposentado (a) & Número Absoluto & Número Relativo (\%) \\
\hline Não & 18 & 12,67 \\
\hline Sim, por tempo de serviço & 71 & 50 \\
\hline Sim, por invalidez & 5 & 3,52 \\
\hline Sim, por idade & 41 & 28,87 \\
\hline Não respondeu & 07 & 4,92 \\
\hline Valor $^{(1)}$ & Número Absoluto & Número Relativo (\%) \\
\hline $\mathrm{R} \$ 937,00$ & 50 & 35,21 \\
\hline $\mathrm{R} \$ 937,00$ a $1.200,00$ & 05 & 3,52 \\
\hline $\mathrm{R} \$ 1.200,00$ a $1.500,00$ & 05 & 3,52 \\
\hline $\mathrm{R} \$ 1.500,00$ ou mais & 38 & 26,76 \\
\hline Não respondeu & 26 & 18,30 \\
\hline
\end{tabular}

'Salário mínimo nacional na época das entrevistas (2017). Fonte: Dados coletados através da pesquisa do autor (GREGORY, 2017).

No que diz respeito à amostra de dados, alusiva à média de idade, 35,91\% estão entre 60 e 65 anos, demonstrando que ao correlacionar o percentual de idosos com o ensino superior completo, somente $16,90 \%$ finalizaram, reafirmando os dados nacionais, em que existem apenas 24 mil pessoas formadas com idade superior a 60 anos. Segundo o último censo 2016 do Instituto Nacional de Estudos e Pesquisas Educacionais, este percentual pode ter influência do local de nascimento, em que 71\% nasceram e cresceram na zona rural (Brasil, 2017).

A partir deste fato, surge a compreensão do meio cultural e social, onde $96 \%$ dos entrevistados consideram-se da cor branca. Destes, 59,15\% migraram do estado do Rio Grande do Sul para Santa Catarina, provocando diversas transformações, o que claramente está ligada a colonização alemã do Sul do Brasil. Por mais que seja conhecida pelo seu conservadorismo das 
fontes culturais, essa influência demonstra que na velhice ainda á tempo para estudar e estar na universidade, que é reconhecida pela sociedade como um local frequentado por jovens.

As oportunidades de formação no ensino superior a 40 anos atrás estavam apoiadas em conceitos díspares ao que conhecemos hoje, o que pode justificar em partes a grande procura de novas experiências por parte dos idosos, pois é uma chance de findar alguns objetivos do passado. O fator ensino superior e velhice pode ter várias relações, sendo a principal o fato da educação ser uma fonte de acesso livre, independente da faixa etária, sendo portanto, um direito de todos (Costa, 2012).

Considerando ainda o exposto na tabela 01 , verifica-se que $50 \%$ dos entrevistados são aposentados por tempo de serviço, sendo que, 35,21\% recebem até $R \$ 937,00$. Já ao analisarmos o critério sócio econômico dos participantes, predominaram as classes B2, com 37,32\% e C1, aprestando $28,16 \%$ dos entrevistados. Estes dados foram obtidos através das variáveis ligadas ao grau de escolaridade do chefe da família, acesso aos serviços públicos e a quantidades de itens domiciliares, como aparelhos eletrônicos, automóveis, empregadas e demais afins (considerando que o salário mínimo da época das entrevistas era $R \$ 937,00)$ (Brasil, 2017).

Em consonância com os dados obtidos, o valor cobrado pelas instituições para gerar acesso à universidade da terceira idade, chega a $30 \%$ do valor recebido ou reconhecido como aposentaria, motivando e dando oportunidades a essa população, que necessita de compreensão das famílias e da sociedade. Sabemos que a velhice chega para todos independente do contexto social e saúde de cada pessoa, dessa forma, verifica-se que a maioria dos idosos entrevistados mora acompanhada de outra pessoa, destes $56 \%$ são casados, tornando-se uma característica predominante nessa faixa etária (Tavares, 2008).

A amostragem demonstra ainda, que perante a divisão da composição familiar, $59 \%$ moram com seus conjugues, $15 \%$ com seus filhos, e os demais com seus namorados, irmãs, mãe e netos, onde $80 \%$ gostam de estar morando com as pessoas com quem residem na atualidade, e $4 \%$ não gostam. Esse resultado é importante para entender o meio familiar desses idosos, sendo relevante para verificarmos a saúde emocional e social dessa população.

Perante o Conselho Federal de Psicologia (2019) a convivência familiar durante o envelhecimento, contribui para a assistência e apoio, para as necessidades dos idosos, que pode ser fundamental para o equilíbrio psicológico, onde as relações afetivas da vida familiar fortalecem a diminuição de frustrações, gerando afeto, prazer e alegria perante o convívio familiar.

Em relação às condições de saúde e hábitos da vida em geral, 57\% consideram boa, 25\% ótima, $17 \%$ regular, $1 \%$ ruim e mais $1 \%$ péssima, apesar da percepção da saúde dos participantes estarem em sua maioria boa, estes resultados sinalizam que é importante acompanhar seus hábitos para identificar possíveis problemas, bem como, as fontes de desvalia perante os idosos que interpretaram esse quesito como ruim e péssimo. Para isso, é preciso adquirir uma mentalidade preventiva e promover adequadamente melhorias para a saúde do idoso. É fundamental a evolução 
e disseminação do conhecimento sobre o envelhecimento para todas as faixas etárias, para que todos consigam detectar e eliminar situações que possam pôr em risco a qualidade de vida presente ou futura (Veras e Caldas, 2004).

Em contrapartida com as considerações expostas acima, as associações entre medicamentos, qualidade de vida, capacidade funcional e cognitiva, mostram que $67 \%$ dos idosos usam algum tipo de medicação, sendo que destes, 70\% foram receitados por médicos. Apesar de ingestão medicamentosa ser alta entre os participantes, apenas $2 \%$ dos participantes são considerados polimedicados.

Ainda, em relação às formas de acesso aos medicamentos, 37\% adquirem através do SUS, e $32 \%$ por compras particulares, sendo que nos últimos 12 meses $39 \%$ dos idosos quando estavam doentes ou necessitaram de consulta médica procuraram as unidades básicas de saúde.

Ao questionarmos os entrevistados sobre doenças e diagnósticos, os resultados demostraram que as cinco principais doenças diagnosticadas são: problemas na coluna, pressão alta, problemas para dormir, osteoporose e má circulação (varizes), sendo estas consideradas doenças crônicas não transmissíveis. Comparando com os dados do teste Whoogol bref resultou em uma média de 4,15, que é ótimo quando analisado dentro da escala, no entanto controversa, pois os mesmos elencam vários diagnósticos, o que nos deixa questionamentos acerca da real qualidade de vida apresentada, podendo ser até mesmo resultado do uso continuo de fármacos.

Ao que se refere às alterações de humor, a depressão é o transtorno que acomete grande parte da população idosa, e que geralmente pode ser confundida com a fase de vida em que se encontram, agravando os quadros de patologias crônicas já existentes. Com difícil diagnóstico os números obtidos da análise, demonstram que 13\% dos 142 entrevistados são diagnosticados com o transtorno, e somente para $7 \%$ ela interfere na vida cotidiana. Já equivalentes à análise de Yesavagea, 7\% estão dentro da escala de 6 a 10, que sugere com depressão leve, sendo que o restante dos entrevistados, não apresentaram quadro depressivo. Além da depressão ser uma doença grave, ela pode gerar agravantes incapacitantes nas atividades mais simples da vida diária, o que indica que quando mais breve o diagnóstico, maior será a probabilidade de tratamento (Gullich et al., 2016).

Sendo uma das maiores preocupações na velhice, a independência é algo que pode ser prejudicada com o quadro patológico citado acima, ligado a esse quesito, a Política Nacional de Saúde da Pessoa Idosa (PNSPI) de 2006, colocou que a saúde de um idoso é classificada pelo grau de autonomia e independência. Sendo que, independentes, são pessoas que apesar de ser ou não portadores de alguma patologia são capazes de realizar sem ajuda, atividades da vida diária. Já os indivíduos que apresentam dificuldades em alguns contextos, são classificados como idosos com potencial para desenvolver fragilidades, e frágeis, são considerados os indivíduos que se encontram acamados, incapacitados de exercer as funções do dia a dia (Brasil, 2006). 
Os resultados obtidos através da escala Lawton, retratam que $50 \%$ possuem dependência parcial, e outros $46 \%$ são independentes. Interligando essas informações para o índice de Barthel, apenas 6\% apresentam dependência leve, enquanto $89 \%$ são totalmente independentes, apresentando um desempenho preocupante, porém, comum em nossa região, onde a maior fonte de renda é a agricultura, e pouco se preocupam com a qualidade de vida da pessoa idosa. Diante desse contexto, uma forma de prevenção da perda cognitiva de idosos é a prática de atividade física regular, adoção de uma dieta equilibrada, estímulo contínuo da atividade cerebral através de interações sociais e atividades intelectuais, como leitura, partidas de xadrez, gamão ou outros jogos (Nordon et al., 2009). A tabela 2 apresenta os tempos e níveis de realização de atividades físicas, que os idosos entrevistados têm praticado.

Tabela 2. Indicador relacionado à frequência e duração de caminhadas, atividades cotidianas que exigem esforços físicos de intensidades moderada e vigorosa.

\begin{tabular}{|c|c|c|}
\hline $\begin{array}{l}\text { Quantas vezes caminhou na } \\
\text { semana }\end{array}$ & Número Absoluto & Número Relativo (\%) \\
\hline 1 dia & 07 & 4,92 \\
\hline 2 a 3 dias & 52 & 36,61 \\
\hline 4 a 5 dias & 25 & 17,60 \\
\hline Todos os dias & 41 & 28,87 \\
\hline Não caminhou & 15 & 10,56 \\
\hline Não respondeu & 02 & 1,40 \\
\hline $\begin{array}{l}\text { Quanto tempo permaneceu } \\
\text { caminhando }\end{array}$ & Número Absoluto & Número Relativo (\%) \\
\hline 10 a 15 minutos & 10 & 7,04 \\
\hline 16 a 30 minutos & 43 & 30,28 \\
\hline 31 a 45 minutos & 13 & 9,15 \\
\hline 1 hora ou mais & 59 & 41,54 \\
\hline Não respondeu & 02 & 1,40 \\
\hline $\begin{array}{l}\text { Quantas vezes realizou atividades } \\
\text { moderadas na semana }\end{array}$ & Número Absoluto & Número Relativo (\%) \\
\hline 1 dia & 22 & 15,49 \\
\hline 2 a 3 dias & 45 & 31,69 \\
\hline 4 a 5 dias & 17 & 11,92 \\
\hline Todos os dias & 20 & 14,08 \\
\hline Não Realiza & 32 & 22,53 \\
\hline Não Respondeu & 06 & 4,22 \\
\hline $\begin{array}{l}\text { Quanto tempo } \\
\text { realizando atividades } \\
\text { moderadas }\end{array}$ & Número Absoluto & Número Relativo (\%) \\
\hline 10 a 15 minutos & 03 & 2,11 \\
\hline 16 a 30 minutos & 12 & 8,45 \\
\hline 31 a 45 minutos & 02 & 1,40 \\
\hline 1 hora ou mais & 85 & 59,85 \\
\hline Não respondeu & 06 & 4,22 \\
\hline
\end{tabular}

Fonte: Dados coletados através da pesquisa do autor (GREGORY, 2017). 
Tabela 2. Continuação.

\begin{tabular}{|c|c|c|}
\hline $\begin{array}{lll}\text { Quantas } & \text { vezes } & \text { realizou } \\
\text { atividades vigorosas na semana }\end{array}$ & Número Absoluto & Número Relativo (\%) \\
\hline $1 \mathrm{dia}$ & 10 & 7,04 \\
\hline 2 a 3 dias & 12 & 8,45 \\
\hline 4 a 5 dias & 02 & 1,40 \\
\hline Todos os dias & 02 & 1,40 \\
\hline Não Realiza & 111 & 78,16 \\
\hline Não Respondeu & 05 & 3,52 \\
\hline $\begin{array}{l}\text { Quanto tempo } \text { permaneceu } \\
\text { realizando atividades vigorosas }\end{array}$ & Número Absoluto & Número Relativo (\%) \\
\hline 10 a 15 minutos & -- & -- \\
\hline 16 a 30 minutos & 02 & 1,40 \\
\hline 31 a 45 minutos & 02 & 1,40 \\
\hline 1 hora ou mais & 19 & 13,38 \\
\hline Não respondeu & 07 & 4,92 \\
\hline
\end{tabular}

Fonte: Dados coletados através da pesquisa do autor (GREGORY, 2017).

Frente aos dados expostos na tabela 2 é possível identificar que $36,61 \%$ dos entrevistados caminham de 2 a 3 vezes por semana, e, 28,87\% praticam atividades todos os dias; combinando a pratica de exercícios com a vida. Estes idosos tendem a aumentar as relações sociais e manutenção da aptidão física, favorecendo um maior preparo, somando também para o bem-estar físico e mental (Shepard, 2003).

Ainda dentro deste parâmetro, o fator tempo é primordial para identificar o nível de disposição para a prática da caminhada. Percebe-se que $41,54 \%$ dos entrevistados dedicam-se mais de uma hora, o que pode influenciar não somente na melhora da autoestima, mas principalmente na autonomia de manutenção da capacidade funcional, que é medida a partir do grau de preservação da condição do indivíduo para realizar atividades diariamente e do grau para desempenhar as atividades instrumentais da vida diária (Machado, 2010).

Ao analisarmos as respostas relativas a avaliação da satisfação com a saúde aferida pelo whoqoll bref, concluiu-se que a média final quanto ao domínio físico foi de 4,03. O que representa um indicativo de que o estimulo de um envelhecimento ativo, pode prevenir doenças, auxiliar na socialização e manter a capacidade cognitiva e funcional da pessoa idosa, de modo que todas alterações, decorrentes do processo de envelhecimento, podem ter seus efeitos minimizados pela adoção de um estilo de vida mais ativo, que pode ser efetivado com a implementação de políticas públicas voltadas a melhorar a qualidade de vida dos idosos (Brasil, 2006).

\section{CONSIDERAÇÕES FINAIS}

A presente pesquisa mostrou que a população de idosos estudada, são portadores de doenças crônicas não transmissíveis, induzindo por isso, a serem polimedicados.

Apresentam apoio familiar, convivendo principalmente com os conjugues e filhos. 
A maioria não apresenta depressão e os poucos que apresentam, é considerada leve.

Quanto à capacidade funcional e cognitiva, possuem comprometimentos menores, que não impossibilitam a realização das principais atividades da vida diária.

Mostram possuir qualidade de vida, apresentando bem-estar físico, psicológico e social, bem como uma boa relação com o meio ambiente onde estão inseridos.

A participação em uma universidade voltada para a terceira idade pode contribuir para a melhoria da qualidade de vida da população idosa. Isso é possível devido a novas aprendizagens que refletem em mudanças de hábitos e comportamentos, refletindo em melhorias e maiores cuidados com a saúde, fazendo com que idosos possuam bem-estar em maiores âmbitos de suas vidas, resultando em um envelhecer saudável, menos medicado e com maior vigor e vontade de viver.

\section{REFERÊNCIAS}

ABEP - Associação Brasileira de Empresas de Pesquisa. Critério de classificação econômica Brasil, 2015. Disponível em: http://www.abep.org/criterio-brasil. Acesso em: 02 jul. 2015.

Aires, M.; Bierhals, C. C. B. K.; Paskulin, L. M. G.; Valer, D. B. O significado de envelhecimento saudável para pessoas idosas vinculadas a grupos educativos. Revista Brasileira de Geriatria e Gerontologia, 18 (4), 809-819, 2015.

Altman, M. O envelhecimento à luz da psicanálise. Jornal de Psicanálise, 44(80), 193-206, 2011.

Araújo, F. C.; Gomes, R.; Nascimento, E. F. Por que os homens buscam menos os serviços de saúde do que as mulheres? As explicações de homens com baixa escolaridade e homens com ensino superior. Revista Saúde Pública, 23(3), 565-574, 2007.

Brasil. Instituto Brasileiro de Geografia e Estatística (IBGE), Agencia de Notícias. Pesquisa Nacional por Amostra de Domicílios Contínua - PNAD Contínua, 2017.

Brasil. Lei n. 10.741, de $1^{\circ}$ de outubro de 2003. Dispõe sobre o Estatuto do Idoso e dá outras providências. Diário Oficial da União, Brasília, 3 out. 2003.

Brasil. Ministério da Saúde. Secretaria de Atenção à Saúde. Departamento de Atenção Básica. Envelhecimento e saúde da pessoa idosa. Departamento de Atenção Básica - Brasília: Ministério da Saúde, 2006.

Carvalho, M. F. C.; Romano-Lieber, N. S.; Bergsten-Mendes, G; Secoli, S. R.; Ribeiro, E.; Lebrão, M. L.; Duarte, Y. A. O. Polifarmácia entre idosos do Município de São Paulo - Estudo SABE. Revista Brasileira de Epidemiologia, 15(4), 817-827, 2012.

Conselho Federal De Psicologia. Envelhecimento e subjetividade: desafios para uma cultura de compromisso social. Brasília, 2008. Disponível em: http://site.cfp.org.br/wpcontent/uploads/2009/05/livro_envelhecimentoFINAL.pdf. Acesso em: 23 de jan. 2019.

Costa, E. M. Gerontodrama: A velhice em cena. Estudos clínicos e psicodramáticos sobre o Envelhecimento e a terceira idade. São Paulo: Ágora Editora, 1998.

Costa, L. Acadêmico idoso no ensino superior: caraterística de altas habilidades/superdotação? Universidade de Santa Maria, RS, 2012. Disponível em: <https://repositorio.ufsm.br/bitstream/handle/1/7003/COSTA\%2C\%20LEANDRA\%20COSTA\%20D A.pdf?sequence=1\&isAllowed=y >. Acesso em: 11 jul. 2018. 
Ferrari, J. F.; Dalacorte, R. R. Uso da Escala de Depressão Geriátrica de Yesavage para avaliar a prevalência de depressão em idosos hospitalizados. Scientia Médica, 17(1), 3-8, 2007.

Ferreira, M. B. C. Prescrição de medicamentos em geriatria. In: FUCHS, F. D.; WANNMACHER, L. Farmacologia clínica: fundamentos da terapêutica racional. 4.ed. Rio de Janeiro: Guanabara Koogan, 2010.

Flores, L. M.; Mengue, S. S. Uso de medicamentos por idosos em região sul do Brasil. Revista Saúde Pública, 39(6), 924-929, 2005.

Fonseca, A. M. Promoção do desenvolvimento psicológico no envelhecimento. Contextos Clínicos, 3(2), 124-131, 2010.

Geib, L. T. C. Determinantes sociais da saúde do idoso. Ciência Saúde Coletiva, (17)1, 123133, 2012.

Gullich, I.; Duro, S. M. S.; Cesar, J. A. Depressão entre idosos: um estudo de base populacional no Sul do Brasil. Revista Brasileira de Epidemiologia, 19(4), 691-701, 2016.

Lawton, M. P. The varieties of well-being. Experimental Aging Research, 9(2), 159-170, 1983.

Lopes, M. E. P. S. A velhice no século XXI: a vida feliz e ainda ativa na melhor idade. Acta Scientiarum. Human and Social Sciences, 34(1), 27-30, 2012.

Machado, F. N. Capacidade e desempenho para a realização das atividades básicas de vida diária: um estudo com idosos dependentes. Minas Gerais, Brasil. 129f. Dissertação (Mestrado em Enfermagem) - Universidade Federal de Minas Gerais, Belo Horizonte. 2010.

Mahoney, F. I.; Barthel, D. W. Functional evaluation: The Barthel Index. Maryland State Medical Journal, 5(14), 61-65, 1965.

Marcon, S. Polimedicação e fatores associados em idosos que frequentam a cidade do idoso de Chapecó/SC. Santa Catarina, Brasil. 112f. Dissertação (Mestrado em Ciências da Saúde) Universidade Comunitária da Região de Chapecó, Chapecó. 2016.

Morais, E. P. Envelhecimento no meio rural: condições de vida, saúde e apoio dos idosos mais velhos de Encruzilhada do Sul - RS. 215 f. Tese (Doutorado em Enfermagem Fundamental) - Escola de Enfermagem de Ribeirão Preto. Universidade de São Paulo, Ribeirão Preto. 2007.

Neves, S. J. F.; Marques, A. P. O.; Leal, M. C. C.; Diniz, A. S.; Medeiros, T. S.; Arruda, I. K. G. Epidemiologia do uso de medicamentos entre idosos em área urbana do Nordeste do Brasil. Revista Saúde Pública, 47(4), 759-768, 2013.

Nordon, D. G.; Guimarães, R. R.; Kozonoe, D. Y.; Mancilha, V. S.; Dias Neto, V. S. Perda Cognitiva em Idosos. Revista da Faculdade de Ciências Médicas de Sorocaba, 11(3), 5-8, 2009.

Papaléo Neto, M. Ciência do envelhecimento: abrangência e termos básicos e objetivos. In: Papaléo Neto, M. Tratado de Gerontologia. 2.ed., São Paulo: Atheneu, 2007.

Parahyba, M. I.; Veras, R.; Melzer, D. Incapacidade funcional entre as mulheres idosas no Brasil. Revista de Saúde Pública, 39(3), 383-391, 2005.

Pereira, M. G. Epidemiologia: teoria e prática. Rio de Janeiro: Guanabara Koogan, 2006.

Ribas, C.; Oliveira, K. R. Perfil dos medicamentos prescritos para idosos em uma Unidade Básica de Saúde do município de ljuí-RS. Revista Brasileira de Geriatria e Gerontologia, 17(1), 99114, 2014. 
Ribeiro, P. C. C.; Yassuda, M. S. Cognição, estilo de vida e qualidade de vida na velhice. In: Neri, A. L. (org.) Qualidade de vida na velhice: enfoque multidisciplinar. Campinas: Editora Alínea, 2007.

Rikli, R. E.; Jones, C. J. Teste de Aptidão Física para Idosos. 1a ed. Barueri: Manole. 2008.

Rosa, T. E. C.; Benício, T. E. C.; D’Aquino, M. H.; Latorre, M. R. D. O.; Ramos, L. R. Fatores determinantes da capacidade funcional entre idosos. Revista Saúde Pública, 37(1), 2003.

Rozenfeld, S.; Fonseca, M. J. M.; Acúrcio, F. A. Drug utilization and polypharmacy among the elderly: a survey in Rio de Janeiro City, Brazil. Revista Panamericana Salud Pública, 23(1), 3443, 2008.

Schneider, R. H.; Irigaray, T. Q. O envelhecimento na atualidade: aspectos cronológicos, biológicos, psicológicos e sociais. Estudos de Psicologia, 25(4), 585-593, 2008.

Secoli, S. R. Polifarmácia: interações e reações adversas no uso de medicamentos por idosos. Revista Brasileira de Enfermagem, 63(1), 136-140, 2010.

Shepard, R. J. Envelhecimento, Atividade Física e Saúde. São Paulo: Phorte, 2003.

Silva, L. R. F. Da velhice à terceira idade: o percurso histórico das identidades atreladas ao processo de envelhecimento. História, Ciências, Saúde, 15(1), 155-168, 2008.

Silva, H. S.; Lima, Â. M. M.; Galhardoni, R. Envelhecimento bem-sucedido e vulnerabilidade em saúde: aproximações e perspectivas. Interface: comunicação, saúde e educação, 14(35), 867-877, 2010.

Silva, A. L.; Ribeiro, A. Q.; Klein, C. H.; Acurcio, F. A. Utilização de medicamentos por idosos brasileiros, de acordo com a faixa etária: um inquérito postal. Cadernos de Saúde Pública, 28(6), 1033-1045, 2012.

Tavares, D. E. A presença do aluno idoso no currículo da universidade contemporânea: uma leitura interdisciplinar. 297f. Tese (Doutorado em Educação: Currículo) Pontifícia Universidade Católica, São Paulo. 2008.

Veras, R. P. Envelhecimento populacional contemporâneo: demandas, desafios e inovações. Revista Saúde Pública, 43(3), 548-554, 2009.

Veras, R. P.; Caldas, C. P. Promovendo a saúde e a cidadania do idoso: o movimento das universidades da terceira idade. Ciência e Saúde coletiva, 9(2), 423-432, 2004.

World Health Organization (WHO). Envelhecimento ativo: uma política de saúde. Brasília: Organização Pan-Americana da Saúde, 2005. 